\title{
A New Function Associated with the Prime Factors of $\left(\begin{array}{l}n \\ k\end{array}\right)$
}

\author{
By E. F. Ecklund, Jr., P. Erdös and J. L. Selfridge
}

\begin{abstract}
Let $g(k)$ denote the least integer $>k+1$ so that all the prime factors of $\left(\begin{array}{c}0(k) \\ k\end{array}\right)$ are greater than $k$. The irregular behavior of $g(k)$ is studied, obtaining the following bounds:

$$
k^{1+c}<g(k)<\exp (k(1+o(1))) \text {. }
$$
\end{abstract}

Numerical values obtained for $g(k)$ with $k \leqq 52$ are listed.

The prime factors of $\left(\begin{array}{l}n \\ k\end{array}\right)$ have been studied a great deal. In a recent paper, Erdös [2] stated several results and unsolved problems on this subject. In this paper, we discuss one of the problems stated there: Denote by $g(k)$ the least integer $>k+1^{*}$ so that all prime factors of $\left(\begin{array}{c}o(k) \\ k\end{array}\right)$ are greater than $k$. Determine or estimate $g(k)$.

The behavior of $g(k)$ is surprisingly irregular. We searched for values of $g(k) \leqq$ 2500000 for $2 \leqq k \leqq 100$; the results of this search are reported in Table 1 . In reviewing Table 1 , we noticed the surprising example $g(28)=284$. This motivated a second search for other such examples with $g(k) \leqq 100000$ and $101 \leqq k \leqq 500$; none were found.

TABLE 1. Values of $g(k) \leqq 2500000$ for $2 \leqq k \leqq 100$

\begin{tabular}{rrrrrrrrrc}
\hline$k$ & $g(k)$ & $k$ & $g(k)$ & $k$ & $g(k)$ & $k$ & $g(k)$ & $k$ & $g(k)$ \\
\hline & & 11 & 47 & 21 & 14871 & 31 & 341087 & 41 & $B$ \\
3 & 6 & 12 & 174 & 22 & 19574 & 32 & 371942 & 42 & 96622 \\
4 & 7 & 13 & 2239 & 23 & 35423 & 33 & 6459 & $43 /$ & $B$ \\
5 & 7 & 14 & 239 & 24 & 193049 & 34 & 69614 & 45 & \\
6 & 23 & 15 & 719 & 25 & 2105 & 35 & 37619 & 46 & 692222 \\
7 & 62 & 16 & 241 & 26 & 36287 & 36 & 152188 & $47 /$ & $B$ \\
8 & 44 & 17 & 5849 & 27 & 1119 & 37 & 152189 & 51 & $B$ \\
9 & 159 & 19 & 2098 & 28 & 284 & 38 & 487343 & 52 & 366847 \\
10 & 46 & 20 & 43196 & 30 & 58782 & 40 & 85741 & 100 & $B$ \\
& \multicolumn{7}{c}{$B: g(k)$ exceeds the search bound of 2500000} & & \\
\hline
\end{tabular}

The following conjectures on $g(k)$ all seem certainly true, and perhaps some of them will not be difficult to prove. First, we conjecture

$$
\begin{aligned}
& \underset{k \rightarrow \infty}{\limsup } g(k+1) / g(k)=\infty \quad \text { and } \\
& \liminf _{k \rightarrow \infty} g(k+1) / g(k)=0 .
\end{aligned}
$$

Received May 7, 1973.

AMS (MOS) subject classifications (1970). Primary $10 \mathrm{H} 15$.

* The condition $g(k)>k+1$ was inserted to avoid the special case $k+1=p$, a prime. 
Also, it seems that $g(k)$ is not of polynomial growth-in other words, for every $n$ and $k>k_{0}(n)$,

$$
g(k)>k^{n}
$$

On the other hand,

$$
\lim _{k \rightarrow \infty} g(k)^{1 / k}=1
$$

certainly seems to hold, and we expect that

$$
g(k)<\exp \left(c_{1} \pi(k)\right)
$$

is true.

We now give lower and upper bounds for $g(k)$. For a lower bound, we show there is an absolute constant $c>0$ such that

$$
g(k)>k^{1+c} \text {. }
$$

We first show that $g(k)>2 k$ (for $k>4$ ) always holds. By definition, $g(k)>k+1$, and $g(k) \neq 2 k$ since $\left(\begin{array}{c}2 k \\ k\end{array}\right)$ is always even. Suppose $g(k)=k+t$ with $1<t<k$. We have $\left(\begin{array}{c}k+t \\ k\end{array}\right)=\left(\begin{array}{c}k+t \\ t\end{array}\right)$. Ecklund [1] showed that $\left(\begin{array}{c}k+t \\ t\end{array}\right)$ has a prime factor not exceeding $(k+t) / 2<k$, the only exception being $\left(\begin{array}{l}7 \\ 3\end{array}\right)$ which corresponds to the case $k=4$, $t=3$. Erdös and Selfridge [2, p. 406] proved that if $m \geqq 2 k$, then $\left(\begin{array}{c}m \\ k\end{array}\right)$ always has a prime factor $\left\langle m / k^{c}\right.$, for some absolute constant $c>0$. This immediately implies (6).

Next, we give a very crude upper bound on $g(k)$. Denote by $L_{k}$ the least common multiple of the integers $1,2, \cdots, k$ and put $P_{l}=\prod_{p \leq l} p$. Let $N(k, l)=L_{k} P_{l}$. If $n+1$ is any multiple of $N(k, l)$, then

$$
\left(\begin{array}{l}
n \\
k
\end{array}\right)=\left(\frac{m N(k, l)}{1}-1\right)\left(\frac{m N(k, l)}{2}-1\right) \cdots\left(\frac{m N(k, l)}{k}-1\right)
$$

has no prime factors less than $l$. Thus,

$$
g(k)<N(k, k)=\prod_{p \leq k} p^{\alpha_{p+1}},
$$

where $\alpha_{p}=\left[\log _{p} k\right]$. For $k>k_{0}$, this upper bound can be improved a bit. We show

$$
g(k)<k^{2} L_{k} P_{l} \quad \text { with } l=[6 k / \log k] .
$$

To prove (8), consider the integers $t L_{k} P_{l}-1$ for $1 \leqq t \leqq k^{2}$. We show that, for at least one of these values of $t$,

$$
p \nmid\left(\begin{array}{c}
t L_{k} P_{l}-1 \\
k
\end{array}\right) \text { for every } p \leqq k .
$$

For $p \leqq l$, (9) holds as before. If $l<p \leqq k$,

$$
p \mid\left(\begin{array}{c}
t L_{k} P_{l}-1 \\
k
\end{array}\right)
$$

can only hold if there is a $j, 1 \leqq j \leqq k$, for which

$$
t L_{k} P_{l} \equiv j\left(\bmod p^{\alpha_{p}+1}\right) \text {. }
$$

The number of integers $t$ with $1 \leqq t \leqq k^{2}$, for which (10) holds, is at most 


$$
k\left(\left[k^{2} / p^{2}\right]+1\right), \text { since } \alpha_{p}=1 \text { for } p>l .
$$

Thus, by (10) and (11), the number of integers $t, 1 \leqq t \leqq k$, for which (10) holds for some prime $p, l<p \leqq k$, is at most

$$
\sum_{i<p \leq k} k\left(\left[k^{2} / p^{2}\right]+1\right)<k^{3} \sum_{p>l} 1 / p^{2}+k \pi(k) .
$$

It easily follows from the prime number theorem that, for $k>k_{0}$,

$$
\sum_{p>l} 1 / p^{2}<\frac{2}{l \log l}<\frac{1}{2 k}
$$

From (12) and (13), for $k>k_{0}$, the number of integers $t, 1 \leqq t \leqq k$, for which (10) holds, is less than $k^{2} / 2+k \pi(k)<k^{2}$. Thus, there is a $t \leqq k^{2}$ with (9) holding for every $p \leqq k$. Thus, $g(k)<k^{2} L_{k} P_{l}$ as stated. The value 6 could be replaced by a smaller constant, but we cannot prove $g(k)<L_{k}$, which seems to hold for all $k$.

It is well known that $L_{k}<\exp (k(1+o(1)))$ and $k^{2} P_{l}<\exp (o(k))$. Thus, $g(k)<$ $\exp (k(1+o(1)))$. So $g(k)<L_{k}$ should be achievable.

Department of Mathematical Sciences

Northern Illinois University

DeKalb, Illinois 60115

1. E. F. ECKIUND, JR., "On prime divisors of the binomial coefficient," Pacific J. Math., v. 29, 1969, pp. 267-270. MR 39 \#5465.

2. P. ERDös, "Some problems in number theory," in Computers in Number Theory, Academic Press, London, 1971, pp. 405-414. 